\title{
Community-based rehabilitation service in Chengdu, Southwest China: a cross- sectional general survey
}

\author{
Siliang Chen ${ }^{1}$, Yi Lei ${ }^{2^{*}} \mathbb{D}$, Hua Dai ${ }^{2}$, Jia Wu², Ziyu Yang ${ }^{2}$ and Xiaoyang Liao ${ }^{2 *}$
}

\begin{abstract}
Background: World Health Organization initiated community-based rehabilitation (CBR) in 1978, and by now, it has been an essential process of medical services worldwide. China had strengthened primary health care on building more than 35,000 community health centers (CHCs) in cities, and more than 34,000 township health centers (THCs) in the rural area. Nevertheless, it remains unclear that if these primary health centers could provide optional rehabilitation services for disabilities. And this study aims at evaluating the supply capacity of rehabilitation service in primary health centers of Chengdu, a regional center city of southwest China.

Method: We conducted a general investigation of primary health centers in Chengdu, a city located in southwest China with more than 15 million population. Our investigation covered all of Chengdu's 390 primary health centers from October to November 2016. We researched these primary health centers on basic rehabilitation services, diseases, and rehabilitation equipment quantity and quality, and traditional Chinese medicine (TCM) physiotherapy.

Result: Rehabilitation therapy is available in 88.9\% (337 of 379) of all primary health centers. Meanwhile, CHCs slightly surpass THCs with an available rate of $92.2 \%$ (106 of 115) and $87.5 \%$ (231 of 264), respectively. Traditional Chinese Medicine (TCM) physiotherapy is available in $97.1 \%$ (368 of 379) of all primary health centers, $97.3 \%$ (112 of 115) of CHCs and $97.0 \%$ (256 of 264) of THCs. Quantitative analysis showed that substantial factors which could make an impact on the number of patients per year contain: categories of rehabilitation disease $(P<0.001,95 \%$ confidence interval (Cl) $[-1.571,-0.702])$,number of rehabilitation bed $(P<0.001,95 \% \mathrm{Cl}[-1.249,-0.290])$.

Conclusion: CBR and TCM physiotherapy has become accessible for disabilities in most basic health centers of Chengdu City, whereas, available rate of CBR in THCs is lesser than in CHCs, which suggests an imbalance in primary health service development between rural and urban area. Categories of rehabilitation diseases, and the number of rehabilitation beds constitute co-factors that make an impact on the CBR capacity of basic health centers.
\end{abstract}

Keywords: Community-based rehabilitation, General survey, Primary health center

\footnotetext{
* Correspondence: leiyi111@scu.edu.cn; liaoxiaoyang@sohu.com

${ }^{2}$ International medical center/center of general practice, West China Hospital,

Sichuan University, Chengdu 610041, Sichuan, PR China

Full list of author information is available at the end of the article
}

(c) The Author(s). 2020 Open Access This article is licensed under a Creative Commons Attribution 4.0 International License, which permits use, sharing, adaptation, distribution and reproduction in any medium or format, as long as you give appropriate credit to the original author(s) and the source, provide a link to the Creative Commons licence, and indicate if changes were made. The images or other third party material in this article are included in the article's Creative Commons licence, unless indicated otherwise in a credit line to the material. If material is not included in the article's Creative Commons licence and your intended use is not permitted by statutory regulation or exceeds the permitted use, you will need to obtain permission directly from the copyright holder. To view a copy of this licence, visit http://creativecommons.org/licenses/by/4.0/. The Creative Commons Public Domain Dedication waiver (http://creativecommons.org/publicdomain/zero/1.0/) applies to the data made available in this article, unless otherwise stated in a credit line to the data. 


\section{Background}

Community-based rehabilitation (CBR) has been a widelyaccepted pattern for individuals to get access to convenient, flexible and economic rehabilitation services since its implementation in the 1970s [1]. A strategy called communitybased rehabilitation was launched by the World Health Organization in 1978 to meet the need of rehabilitation services around the world [2]. Since being initiated, CBR has been introduced into more than 90 countries as a rational pattern to deliver rehabilitation services. Statistics show that more than 1 billion people are with disabilities globally. And about 110-190 million adults and 93 million children are experiencing significant difficulties in daily function [3]. The World Health Organization (WHO) set CBR as a critical development direction for disabilities, according to the Global Disability Action Plan [3].

After being introduced into China in the 1980s, rehabilitation services were mainly provided by volunteers in the early stage. However, due to the lack of systematic training for volunteers, CBR service remains in a low quality for many years. With more investment and support from the government, rehabilitation services based on communities began to develop in a rapid speed to alleviate the lack of rehabilitation services for disabilities [4]. Nevertheless, inadequate financial support and lack of rehabilitation physicians have hindered development of CBR services [5].

There are 34,522 CHCs and 36,871 THCs in mainland China by Sep, 2015 [6]. Even though the Chinese government has devoted significant resources in building primary health centers in recent years, rehabilitation services and personnel are still largely in need. In the next 10 years, it is estimated that China required 60,000 rehabilitation physicians, 150,000 rehabilitation therapists and 60,000 nurses to meet the demand [7]. By the end of 2010, China had more than 85 million disabled persons, according to the census [8]. But until 2018, there were only 9036 professional rehabilitation institutions and about 250,000 staff in these institutions, including physicians, nurses and administrative [9], which indicated that most of the disabled persons in mainland China could not get easy access to rehabilitation service. On the patients' side, they prefer to go to hospitals rather than primary health care centers for what they believe in having more professional service [10]. Consequently, the Chinese government initiated the "Healthy China 2030" plan, in which service capacity and scale of primary health care system was set as a focused point to invest [11].

Nevertheless, there are rare data about how widely the primary health centers could cover disabilities who require convenient and economic rehabilitation services. Because there are many challenges during the process of CBR evaluation would hamper investigators to get reliable data. For instance, community health centers in the vast rural area of China are difficult to be evaluated because of the low literacy and the large variety of dialects.
In addition, participants tend to select the midpoint and skip some items because Chinese culture affects their behavior and drives them prefer to be modest and to underestimate their performance [12-14]. They are ashamed to openly discuss and rate their family or organization [15]. Hence, we designed and performed this cross-sectional survey to evaluate the supply capacity of rehabilitation services in primary health centers of Chengdu and find out how we could promote the development of community-based rehabilitation. We purposely conducted this survey among the leaders of primary health centers rather than patients, which may get objective data and minimize bias caused by Chinese patients' inclination to rate their performance and refrain from exaggerating their achievements modestly.

\section{Methods}

\section{Subjects}

To effectively evaluate $\mathrm{CBR}$ in $\mathrm{CHCs}$, we selected Chengdu, the capital of Sichuan province, in southwest China with a land area of $12,390 \mathrm{~km}^{2}$ and a population of 16.33 million by 2018 [16, 17]. This city is the economic and cultural center of western China, and about $34.49 \%$ of the population dwell in rural areas.

And we conducted a general cross-sectional survey of all 390 primary health centers in Chengdu. To reduce the uncertainty caused by the large variety of education levels of patients, the survey questionnaire was undertaken by lead physicians in these community health facilities from October 2016 to November 2016. At the same time, data about these community health facilities was obtained from the Health Commission of the Sichuan Province to verify survey results. In this way, we expected to receive as accurate and objective data as we could.

In summary, there are two critical directions for China to enhance CBR: the first is to improve the scale of CBR service in primary health centers; the second is to improve service quality to attract patients instead of sending them to superior hospitals. To evaluate priamry health centers from these two aspects, we selected data of the number of patients (person-time) in 2015 as a proxy of CBR service capacity. Since this number could directly reflect the size and volume of CBR service. On the other hand, the larger this number was, the more patients the primary health center attracted, which indicated the higher quality of CBR service.

\section{Development of the questionnaire}

The questionnaire was designed according to the policy of $<$ Service quality evaluation guideline for primary care facilities $>$ [18], made by the National health and family planning commission of the people's republic of China in 2016, and modified after consideration of applicability as well as expert judgment. We focused on evaluating 
the basic capabilities of primary care facilities in providing services Thus we mainly collected the following variables:

1. Total number of rehabilitation patients of each primary health facilities in 2015

2. Total number of disabilities in the area under the jurisdiction of each primary health facilities in 2015

3. Categories of rehabilitation diseases

4. Basic conditions: served population, area of the structure, the total number of rehabilitation physicians, physical therapy equipment, cervical and lumbar traction equipment, infrared therapy apparatus, ultrasound therapy apparatus, number of rehabilitation bed, number of therapeutic rehabilitation room.

5. Rehabilitation training: rehabilitation lecture, rehabilitation counseling.

6. Rehabilitation management measures: rehabilitation training plan, rehabilitation-related system, selfinspection.

The questionnaire is available in additional file 1.

\section{Administration of the questionnaire}

An instruction for data quality control was developed for the questionnaire and then distributed by local health bureaus to the leaders of each primary health center. Three hundred ninety lead physicians completed the questionnaires independently and submitted them to the survey team in 2 weeks after receiving them. Then the survey team staff rechecked the collected questionnaires and filled the missing data by interviewing leaders of primary health centers through telephone. At last, the team randomly sent staff to $5 \%$ of the $\mathrm{CHC}$ facilities to validate data received.

\section{Data analysis}

Up to now, qualitative methodologies have dominated the field of evaluations in community-based rehabilitation, but quantitative methods have demonstrated strong potential in capture a better assessment [19]. Therefore, quantitative data analysis was adopted in our crosssectional survey. Data were logged into Epidata by dual investigators and analyzed with SPSS 22.0 (SPSS Inc., Chicago, IL, USA).

Multivariable ordinal logistic regression analysis was used to determine which specific characteristics were independently related to the number of patients per year in CHCs and THCs-among the number of diseases, rehabilitation diseases, area of the structure, rehabilitation equipment, rehabilitation management measures, etc. All significant tests were 2-tailed, and those with a $P$ value $<0.05$ were considered statistically significant. In addition, we also conducted descriptive analysis on the basic condition of CBR service in primary health centers.

\section{Results}

Of all the $390 \mathrm{CHCs}$ this survey covered, 379 primary health centers responded to this survey, which came out with a response rate of $97.2 \%$. After data validation, all 379 feedbacks entered into the analysis.

The first part is the basic status of community rehabilitation and TCM physiotherapy (Table 1.). Rehabilitation service is available in $88.9 \%$ (337 of 379) of all primary health centers, meanwhile, CHCs slightly surpass THCs with an available rate of $92.2 \%$ (106 of 115) and 87.5\% (231 of 264), respectively. Traditional Chinese Medicine (TCM) physiotherapy is available in $97.1 \%$ (368 of 379) of all primary health centers, $97.3 \%$ (112 of 115) of CHCs and $97.0 \%$ (256 of 264) of THCs. And the top 5 diseases of community rehabilitation in descending order are the cervical vertebral disease, lumbar vertebral disease, osteoarthritis, adhesive capsulitis, and stroke sequelae.

Statistics about the basic condition of $\mathrm{CHCs}$ read that the total number of rehabilitation patients in 2015 were more than 2 million person-time. The average number of patients of $\mathrm{CHCs}$ and THCs were 7456.9 and 4762.6 person-time in 2015, respectively. (Table 2.)

To quantitively analyze potential factors that could influence the capacity of $\mathrm{CHCs}$ rehabilitation service, we covert the dependent variable, the number of rehabilitation patients to level variable according to quartile. (Table 3.)

Quantitively analysis indicated that substantial factors which could make an impact on the number of patients per year contain: species of rehabilitation disease $(P<$ $0.001,95 \%$ confidence interval $(\mathrm{CI})[-1.571$, $0.702])$.number of rehabilitation bed $(P<0.001,95 \% \mathrm{CI}$ $[-1.249,-0.290])$ (Table 4.)

Discussion To authors' knowledge, by Sep 2019, this survey is the ever first general survey of rehabilitation capacity of primary health care in China from a literature study, as well as the first analysis of factors that influence rehabilitation service capacity in the primary health system. In the general survey, we found more than $88 \%$ of CHCs and THCs involved in this survey have been well equipped to provide rehabilitation

Table 1 The status of community rehabilitation and TCM physiotherapy

\begin{tabular}{|c|c|c|c|c|}
\hline & \multicolumn{2}{|c|}{ urban community $(N=115)$} & \multicolumn{2}{|c|}{$\begin{array}{l}\text { rural community } \\
(N=264)\end{array}$} \\
\hline & yes & no & yes & no \\
\hline Rehabilitation & 106 & 9 & 231 & 33 \\
\hline TCM physiotherapy & 112 & 3 & 256 & 8 \\
\hline both & 106 & 9 & 231 & 33 \\
\hline
\end{tabular}


Table 2 The basic condition of primary health centers

\begin{tabular}{|c|c|c|c|c|c|}
\hline & \multicolumn{5}{|c|}{ primary health centers } \\
\hline & $\begin{array}{l}\text { Total of CHCs } \\
(N=115)\end{array}$ & Average of $\mathrm{CHCs}$ & $\begin{array}{l}\text { Total of THCs } \\
(N=264)\end{array}$ & Average of THCs & $\begin{array}{l}\text { Total } \\
(N=379)\end{array}$ \\
\hline Number of patients ${ }^{\mathrm{a}}$ (person-time)(KF01) & $857544^{e}$ & 7456.9 & $1,257,323$ & 4762.6 & $2,114,867$ \\
\hline Number of disabilities ${ }^{\mathrm{b}}$ (KF05) & 69,037 & 600.3 & 156,438 & 592.6 & 225,475 \\
\hline Number of disabilities with health record (KF06) & 47,007 & 408.8 & 109,166 & 413.5 & 156,173 \\
\hline \multicolumn{6}{|l|}{ Basic condition } \\
\hline Building area ${ }^{\mathrm{d}}(\mathrm{RYO2})$ (square meter) & $376,090.85$ & 3270.4 & $893,922.37$ & 3386.1 & $1,270,013.22$ \\
\hline Number of rehabilitation physicians (RY08) & 215 & 1.9 & 323 & 1.2 & 538 \\
\hline \multicolumn{6}{|l|}{ Rehabilitation equipment } \\
\hline Cervical and lumbar traction equipment (KF09) & 107 & 0.9 & 243 & 0.9 & 350 \\
\hline Infra-red ray therapy apparatus & 93 & 0.8 & 188 & 0.7 & 281 \\
\hline Ultrasound therapy equipment & 60 & 0.5 & 93 & 0.4 & 153 \\
\hline Rehabilitation beds (KF02) & 1274 & 11.1 & 2270 & 8.6 & 3544 \\
\hline Number of rehabilitation therapeutic room & 290 & 2.5 & 495 & 1.9 & 785 \\
\hline \multicolumn{6}{|l|}{ Rehabilitation training } \\
\hline Rehabilitation lecturef(times)(KF07) & 847 & 7.4 & 1381 & 5.2 & 2228 \\
\hline Rehabilitation counseling ${ }^{f}$ (times)(KF08) & 21,748 & 189.1 & 9914 & 37.6 & 31,662 \\
\hline \multicolumn{6}{|l|}{ Rehabilitation management measures } \\
\hline rehabilitation training plan (number of launch) ${ }^{9}$ (KF12) & 95 & 0.8 & 189 & 0.7 & 284 \\
\hline Rehabilitation related system (number of launch) (KF13) & 104 & 0.9 & 205 & 0.8 & 309 \\
\hline Self-inspection (number of launch) (KF14) & 86 & 0.7 & 179 & 0.7 & 265 \\
\hline
\end{tabular}

${ }^{\text {a }}$ Number of patients: number of all patients received CBR service in 2015, counted in person-time

${ }^{b}$.Number of disabilities: total number of disabilities in the service area

'.Number of disabilities with health record: total number of disabilities with health documents in primary health centers and visit for CBR regularly in the service area

${ }^{d}$.Building area: total building area of primary health centers

${ }^{\mathrm{e}}$.This number indicates the total number of patients in all $115 \mathrm{CHCs}$

${ }^{f}$.Rehabilitation lecture and counseling in 2015

${ }^{g}$.Number of launch: total number of primary health centers launching this measure

services. But, the imbalance between urban and rural community health centers remains prominent, even though the government has made efforts to minimize the gap. For nearly the same average number of disabilities (600.3 of CHCs, 592.6 of THCs), CHCs' number of patients is 1.56 times as THCs' (7456.9 vs 4762.6$)$. Compared to THCs, CHCs obviously equipped with higherquality equipment, more rehabilitation doctors (1.9 vs 1.2) and more rehabilitation counseling (189.1 vs 37.6). Reasons that make this kind of gap between urban and rural may be various, such as economic level, physician attraction, transportation facilities, and citizens' health awareness. Consequently, this phenomenon of the

Table 3 Conversion of the number of rehabilitation patients

\begin{tabular}{ll}
\hline Variable & Classification criteria \\
\hline Number of patients (person-time/year) & $1:<378 ;$ \\
$2:>=378$ AND $<2375 ;$ \\
$3:>=2375$ AND $<7255 ;$ \\
$4:>=7255$ \\
\hline
\end{tabular}

urban-rural imbalance inspires the government and community to shift more financial support and health education to the rural area to improve the rehabilitation capacity of primary health centers there.

As to factors that were analyzed by multivariable ordinary logistic regression, categories of rehabilitation diseases and the number of rehabilitation bed are considered statistically significant. However, we should not ignore the number of rehabilitation physicians as a potential factor even though it is not statistically significant $(P=0.05,95 \% \mathrm{CI}[-0.882,0.005])$. For instance, $\beta$ value of categories of rehabilitation diseases is -1.136 , which means primary health centers house more than 9 categories of rehabilitation diseases would attract a larger number of patients, comparing to those with less than 9. It indicates that increase the types of rehabilitation diseases would improve CBR service capacity, which should be considered during the planning of the primary health care system. Following the same logic, increasing the number of rehabilitation beds and physicians should also be included in the plan when possible. 
Table 4 Multivariable ordinary logistic regression analysis on the number of patients of primary health centers in Chengdu

\begin{tabular}{|c|c|c|c|c|c|c|}
\hline & & \multirow[t]{2}{*}{$\beta$} & \multirow[t]{2}{*}{ Wald } & \multirow[t]{2}{*}{$P$} & \multicolumn{2}{|c|}{ 95\% Confidence Interval } \\
\hline & & & & & Lower limit & Higher limit \\
\hline \multirow[t]{4}{*}{ Number of disabilities (KF05) } & $<248$ & -0.032 & 0.011 & 0.92 & -0.630 & 0.565 \\
\hline & $>=248$ AND $<486$ & 0.174 & 0.300 & 0.58 & -0.447 & 0.794 \\
\hline & $>=486$ AND $<840$ & 0.144 & 0.219 & 0.64 & -0.460 & 0.748 \\
\hline & $>=840$ & reference & & & & \\
\hline \multirow[t]{2}{*}{ Rehabilitation diseases (KF03) } & $<9$ & -1.136 & 26.242 & 0.00 & -1.571 & -0.702 \\
\hline & $>=9$ & reference & & & & \\
\hline \multirow[t]{4}{*}{ Served population ${ }^{\mathrm{a}}$ (RY01)(10 thousand) } & $<1.8$ & -0.561 & 2.827 & 0.09 & -1.216 & 0.093 \\
\hline & $>=1.8$ AND $<3$ & -0.628 & 4.646 & 0.03 & -1.198 & -0.057 \\
\hline & $>=3$ AND $<5.94$ & -0.194 & 0.468 & 0.49 & -0.750 & 0.362 \\
\hline & $>=5.94$ & reference & & & & \\
\hline \multirow[t]{4}{*}{ Building structure (RY02)(square meter) } & $<1600$ & -0.687 & 3.816 & 0.05 & -1.376 & 0.002 \\
\hline & $>=1600$ AND $<2628.4$ & 0.157 & 0.276 & 0.60 & -0.428 & 0.741 \\
\hline & $>=2628.4$ AND 4296 & -0.297 & 1.091 & 0.30 & -0.855 & 0.260 \\
\hline & $>=4296$ & reference & & & & \\
\hline \multirow[t]{2}{*}{ Number of rehabilitation physicians (RY08) } & $<=1$ & -0.438 & 3.761 & 0.05 & -0.882 & 0.005 \\
\hline & $>1$ & reference & & & & \\
\hline \multirow[t]{2}{*}{ Cervical and lumbar traction equipment (KF09) } & Yes & -1.047 & 1.352 & 0.25 & -2.811 & 0.718 \\
\hline & No & reference & & & & \\
\hline \multirow[t]{2}{*}{ Infra-red ray therapy apparatus } & No & 0.016 & 0.004 & 0.95 & -0.507 & 0.540 \\
\hline & Yes & reference & & & & \\
\hline \multirow{2}{*}{$\begin{array}{l}\text { ultrasound therapy equipment } \\
\text { (KF11) }\end{array}$} & No & -0.196 & 0.758 & 0.38 & -0.638 & 0.245 \\
\hline & Yes & reference & & & & \\
\hline Rehabilitation bed & $<=7$ & -0.770 & 9.908 & 0.00 & -1.249 & -0.290 \\
\hline (KF02) & $>7$ & reference & & & & \\
\hline \multirow[t]{2}{*}{ Number of rehabilitation therapeutic room (KS0301) } & $<=2$ & -0.148 & 0.389 & 0.53 & -0.614 & 0.317 \\
\hline & $>2$ & reference & & & & \\
\hline \multirow[t]{2}{*}{ rehabilitation training plan (KF12) } & No & -0.289 & 0.848 & 0.36 & -0.905 & 0.326 \\
\hline & Yes & reference & & & & \\
\hline \multirow[t]{2}{*}{ Rehabilitation self-examination (KF14) } & No & 0.390 & 1.943 & 0.16 & -0.158 & 0.939 \\
\hline & Yes & reference & & & & \\
\hline
\end{tabular}

${ }^{a}$ Served population: number of all inhabitants in the service area

Since 2009, the Chinese government has been putting a large number of resources to improve its primary health care system. For example, in 2014, the expenditure on primary care reached $¥ 110$ billion [20]. In less than 10 years, the infrastructures and facilities of the $\mathrm{CHCs}$ and THCs were significantly enhanced [21].

Along with the giant leap in the primary health care system under a powerful and effective push from China government on medical services, the rehabilitation capacity of these primary health centers also advanced in both hardware and software. However, in the southwest of China, there are dozens of regions troubling in short of appropriate medical service, and inevitable lack of affordable and convenient rehabilitation services, because of poverty, inadequate transportation and lack of health awareness. Chengdu, as the central city of southwest China, has a powerful and comprehensive influence on regional development in every aspect, including economy, education, culture, technology, and medicine. The outcome of our study, therefor, may be representative to entire southwest China rather than a city.

Due to poor education and training systems on general practitioners, rehabilitation services could not be well delivered to disabilities, even though $\mathrm{CHCs}$ have been equipped with the newest equipment and technology. Hence, creating an optimal general practitioner training system would be another driver that could further improve rehabilitation service capacity in the primary health system. 


\section{Limitation}

In this survey covering all 390 basic primary health centers of Chengdu city, we eventually received 379 intact replies that could be used in analysis. Other 11 primary centers were ruled out from analysis due to incomplete data, which may lead to unknown bias in the results. Another limitation is that we selected the number of patients as a proxy of CBR service, which we think could reflect the scale and service quality of a primary health center. Patients did not know exactly how a primary health center is, they would select which is better according to their experience and impression during accepting CBR service. Hence, patients' subjective judgement decides whether to accept CBR service in $\mathrm{CHCs}$ and then has an influence on our results.

\section{Conclusion}

Rehabilitation service capacity of primary health facilities in Chengdu took a giant leap in the past 20 years and reached a relatively high level in both quantity and quality. However, there are still many flaws and shorts during the rapid development of the primary health system and rehabilitation service. For instance, the imbalance between CHCs and THCs is one of the challenges that need to be addressed. Our study shows that the government could take measures directly correlated with rehabilitation service capacity to improve CBR service capacity, such as house more categories of rehabilitation diseases in the center, train more rehabilitation physicians and augment the number of rehabilitation beds.

\section{Additional Files}

Additional File 1. Self-evaluation form of basic medical service capacity of primary medical institutions in Chengdu

\section{Abbreviations \\ CBR: Community-based rehabilitation; $\mathrm{CHC}$ : Community health center; THC: Town health center; TCM: Traditional Chinese medicine; Cl: Confidence} interval

\section{Acknowledgments}

We would like to thank all the CHCs and THCs who participated in the survey. We would also like to thank the generous support of the Chengdu health and family planning commission.

\section{Authors' contributions}

SC analyzed and interpreted survey data and was a major contributor in writing the manuscript. $Y L$ and $X L$ design of the work. $H D$ and $Z Y$ collected data and contributed to the analysis. JW contributed to writing the manuscript. All authors have read and approved the manuscript.

\section{Funding}

This study was supported by a fund of Chengdu Municipal Commission of Health and Family Planning (project no. 0040205502002) and The National Key Research and Development Program of China (grant numbers 2017YFC0907303)

Chengdu Municipal Commission of Health and Family Planning authorized this survey and provided financial support for this research.

\section{Availability of data and materials}

The datasets used and analyzed during the current study are available from the corresponding author on reasonable request.

\section{Ethics approval and consent to participate}

This survey was authorized by the Chengdu Municipal Health Commission $(\mathrm{CMHC})$. And $\mathrm{CMHC}$ signed a consent document to authorize us to use the information of the survey and information in the database. All primary health centers signed consent documents to be included in this general survey. $\mathrm{CMHC}$ initiated this general survey and accessed these information (not including patients' privacy) under the law of <Regulation on the Administration of Medical Institutions > of People's Republic of China. CMHC was a certified institution to check ethics issues under the law of $<$ Measures for the Ethical Review of Biomedical Research Involving Humans> of People's Republic of China.

\section{Consent for publication}

Not applicable.

\section{Competing interests}

The authors declare that they have no competing interests.

\section{Author details}

${ }^{1}$ West China School of Medicine, Sichuan University, Chengdu 610041, Sichuan, PR China. ${ }^{2}$ International medical center/center of general practice, West China Hospital, Sichuan University, Chengdu 610041, Sichuan, PR China.

Received: 13 November 2019 Accepted: 28 June 2020

Published online: 08 July 2020

\section{References}

1. Lightfoot E. Community-based rehabilitation: a rapidly growing method for supporting people with disabilities. Int Soc Work. 2004;47(4):455-68.

2. World Health Organization. From Alma Ata to the year 2000 : reflections at the midpoint. 1988.

3. World Health Organization. WHO global diability action plan 2014-2021. p. 2015.

4. Ran C, Wen S, Yonghe W, Honglu M. A glimpse of community-based rehabilitation in China. Disabil Rehabil. 1992;14(2):103-7.

5. Hampton N. An evolving rehabilitation service delivery system in the People's Republic of China. J Rehabil. 2001;67(3):20.

6. National Health Commission of the People's Republic of China. Number of medical institutions of mainland China 2016 [Available from: http://www. gov.cn/guoqing/2016-05/13/content_5073060.htm. Accessed 18 Mar 2020.

7. Li J, Li LSW. Development of rehabilitation in China. Phys Med Rehabil Clin N Am. 2019;30(4):769-73.

8. China Disabled Persons' Federation. Number of disabled persons in China by the end of 20102012 [Available from: http://www.cdpf.org.cn/sjzx/ cjrgk/201206/t20120626 387581.shtml. Accessed 18 Mar 2020.

9. China Disabled Persons' Federation. Bulletin of statistics on development of disabled persons in 2018. 2019.

10. Li X, Lu J, Hu S, Cheng KK, De Maeseneer J, Meng Q, et al. The primary health-care system in China. Lancet (London, England). 2017;390(10112): 2584-94.

11. CPC Central Commitee, State Council. The plan for "Healthy China 2030" 2016 [Available from: http://www.gov.cn/zhengce/2016-10/25/ content_5124174.htm. Accessed 18 Mar 2020.

12. Chen C, Lee S, Stevenson HW. Response style and cross-cultural comparisons of rating scales among east Asian and north American students. Psychol Sci. 1995:6(3):170-5.

13. Lee JW, Jones PS, Mineyama Y, Zhang XE. Cultural differences in responses to a likert scale. Res Nurs Health. 2002;25(4):295-306.

14. Farh J, Dobbins $\mathrm{GH}$, Cheng B. Cultural relativity in action: a comparison of selfratings made by Chinese and U.S. workers. Pers Psychol. 1991:44(1):129-47.

15. Shek DTL. The Chinese version of the self-report family inventory: does culture make a difference? Res Soc Work Pract. 1998;8(3):315-29.

16. Chengdu bureau of Statistics. Land area of Chengdu city 2004 [Available from: http://www.cdstats.chengdu.gov.cn/htm/detail_1524.html. Accessed 18 Mar 2020.

17. Chengdu bureau of Statistics. Population of Chengdu by 20182019 [Available from: http://www.cdstats.chengdu.gov.cn/htm/detail_144653. html. Accessed 18 Mar 2020. 
18. National Health Commission of the People's Republic of China. Service quality evaluation guideline for primary health center 2016 [Available from: http://www.nhc.gov.cn/xxgk/pages/viewdocument.jsp?dispatchDate= \&staticUrl=/jws/s2910/201607/4e1 cf3ddc2fc4c68aac2a9f3ffefca77.

shtml\&wenhao=\%E5\%9B\%BD\%E5\%8D\%AB\%E5\%9F\%BA\%E5\%B1\%82\%E7\% A4\%BE\%E5\%8D\%AB\%E4\%BE\%BF\%E5\%87\%BD\%E3\%80\%942016\%E3\%80\% 9565\%E5\%8F\%B7\&utitle=\%E5\%9B\%BD\%E5\%AE\%B6\%E5\%8D\%AB\%E7\%94\% 9F\%E8\%AE\%A1\%E7\%94\%9F\%E5\%A7\%94\%E5\%9F\%BA\%Е5\%B1\%82\%Е5\% 8D\%AB\%E7\%94\%9F\%E5\%8F\%B8\%E5\%85\%B3\%E4\%BA\%8E\%E5\%BC\%80\% E5\%B1\%95\%E7\%A4\%BE\%E5\%8C\%BA\%E5\%8D\%AB\%E7\%94\%9F\%E6\%9C\% 8D\%E5\%8A\%A1\%E4\%B8\%AD\%E5\%BF\%83\%E5\%8C\%BB\%E7\%96\%97\%Е5\% 8D\%AB\%E7\%94\%9F\%E6\%9C\%8D\%E5\%8A\%A1\%E8\%83\%BD\%E5\%8A\%9B\% E8\%B0\%83\%E6\%9F\%A5\%E7\%9A\%84\%E5\%87\%BD\&topictype=\&topic= \&publishedOrg=\%E5\%9F\%BA\%E5\%B1\%82\%E5\%8D\%AB\%E7\%94\%9F\%E5\% 8F\%B8\&indexNum=000013610/2016-00138\&manuscriptld=4e1 cf3ddc2fc4c6 8aac2a9f3ffefca77. Accessed 18 Mar 2020.

19. Grandisson M, Hébert M, Thibeault R. A systematic review on how to conduct evaluations in community-based rehabilitation. Disabil Rehabil. 2014;36(4):265-75.

20. National Health Commission of the People's Republic of China. China Health and Family Planning Statistics Yearbook 2015 Beijing: Peking Union Medical College Press; 2015 [Available from: http://tongji.cnki.net/kns55/ Navi/YearBook.aspx?id=N2015110062\&floor=1. Accessed 18 Mar 2020.

21. Wang HH, Wang JJ, Wong SY, Wong MC, Mercer SW, Griffiths SM. The development of urban community health centres for strengthening primary care in China: a systematic literature review. Br Med Bull. 2015;116:139-53.

\section{Publisher's Note}

Springer Nature remains neutral with regard to jurisdictional claims in published maps and institutional affiliations.

Ready to submit your research? Choose BMC and benefit from:

- fast, convenient online submission

- thorough peer review by experienced researchers in your field

- rapid publication on acceptance

- support for research data, including large and complex data types

- gold Open Access which fosters wider collaboration and increased citations

- maximum visibility for your research: over $100 \mathrm{M}$ website views per year

At $\mathrm{BMC}$, research is always in progress.

Learn more biomedcentral.com/submissions 